\title{
Finite-Time Control of One Dimensional Crowd Evacuation System
}

\author{
Wei Qin (D, , ${ }^{1,2}$ Baotong Cui, ${ }^{1,2}$ and Zhengxian Jiang $\mathbb{D}^{3}$ \\ ${ }^{1}$ Key Laboratory of Advanced Process Control for Light Industry (Ministry of Education), Jiangnan University, Wuxi 214122, China \\ ${ }^{2}$ School of IoT Engineering, Jiangnan University, Wuxi 214122, China \\ ${ }^{3}$ School of Science, Jiangnan University, Wuxi 214122, China
}

Correspondence should be addressed to Wei Qin; weiqin@vip.jiangnan.edu.cn

Received 26 February 2019; Revised 18 June 2019; Accepted 3 July 2019; Published 5 August 2019

Academic Editor: Eneko Osaba

Copyright (C) 2019 Wei Qin et al. This is an open access article distributed under the Creative Commons Attribution License, which permits unrestricted use, distribution, and reproduction in any medium, provided the original work is properly cited.

\begin{abstract}
This paper pertains to the study of finite-time control of one dimensional crowd evacuation system. Benefiting from the research of fluid dynamics and vehicle traffic, a one dimensional crowd evacuation system is constructed, whose density-velocity relationship is represented by a diffusion model. In order to deal with the nondirectionality of crowd movement, the free flow speed is chosen as a control variable. Since the control variable is included in a partial derivative, it increases the difficulty of designing the controller. In this paper, finite-time controller is designed, which not only guarantees the effective evacuation, but also obtains the estimation of evacuation time. Then, finite-time tracking problem is solved, which makes the density converge to a given density. Finally, numerical examples illustrate the effectiveness of the controllers.
\end{abstract}

\section{Introduction}

In everyday life, crowds would gather in many places, for example, subway stations, stadiums, and cinemas. Effective measures should be taken to ensure the safety and comfort of pedestrians, so how to evacuate people when emergencies occur has been a challenging job. In the early 1990s, the International Conference on Engineering for Crowd Safety [1] has shown the importance of this topic. Physicists, sociologists, psychologists, computer scientists, and traffic scientists have been conducting depth-going researches on this topic from their research fields.

Modeling the crowd dynamics is the primary task of crowd evacuation, but due to the complexity and uncertainty of crowd dynamics, it is very difficult to build a model that suits all situations. Therefore, various models have been developed, such as the continuum model, the network-based model [2], agent-based models [3, 4], game-theoretic models [5], cellular automata models [6,7], and the fractional model [8].

In this paper, the continuum model, a macroscopic simulation model, is recommended. At medium and high densities, the motion of pedestrian crowds shows some striking analogies with the motion of fluids [9], so the theory describing fluid dynamics is introduced to describe pedestrian dynamics. Pedestrians are treated as a collection rather than individuals. Average density and velocity at a given location are proposed to describe the crowd dynamics. Based on three hypotheses, a first-order continuum model was developed to describe the pedestrian dynamics in [10]. Huang et al. [11] provided an efficient method to solve Hughes' model. Appert-Rolland et al. [12] extended a macroscopic vehicle traffic model to pedestrian traffic. The cell transmission approach and the continuum approach were combined in [13] to predict densities and travel times. Some continuum models were compared by making use of numerical method in [14]. All above focus on modeling the pedestrian dynamics in different situations, but few literatures present strategies for controlling the crowd dynamics. Wadoo [15] designed advective, diffusive, and advectivediffusive controllers for crowd dynamics in one dimension. Sliding mode control method was applied to crowd dynamic models for the synthesis of robust controllers in [16]. Dong et al. [17] designed feedback control law for two dimensional 
crowd model. Robin, Neumann, and Dirichlet boundary control laws are designed for a disturbed crowd evacuation system in [18].

In this paper, the problem of finite-time evacuation is studied. In some special circumstances, such as fires and terrorist attacks, pedestrians need to be evacuated as soon as possible. Evacuation strategy cannot be used until its effectiveness is tested, because the cost involves not only property damage but also pedestrian injury or death. Therefore, it is particularly important to estimate the evacuation time. The estimated evacuation time can be used to evaluate the effectiveness of the evacuation strategy, and the corresponding evacuation strategy can be adjusted to minimizing the loss. Benefiting from Orlov's research [19], finite-time controller is designed to evacuate pedestrians in finite time, and then the finite-time control method is extended to deal with the tracking problem which makes the crowd density follow a given reference density. Some of the latest research on advanced control, such as adaptive control [20-22], guaranteed cost control [23], $H_{\infty}$ control [24], and tracking control [25, 26], gave us inspiration in the design of the controller. The control and stability problem are formulated directly in the framework of a distributed model of partial differential equations (PDEs), which can avoid errors introduced by spatial discretization. The development of hardware technology has made distributed sensors and actuators realistic, but we mainly explore the crowd management strategies on theoretical interest in this paper, and the implementation needs further study in the future.

The rest of this paper is organized as follows. One dimensional crowd evacuation dynamic is modeled in Section 2. Section 3 presents a feedback controller to evacuate pedestrians in finite time. Section 4 designs a finite-time controller to make the crowd density profile track a reference density. Simulation examples are given in Section 5 to illustrate the effectiveness of the controllers. Conclusions and future work are discussed in Section 6.

Notation. The notation is used throughout the paper. $H^{2}(0, L)$ denotes the infinite-dimensional Hilbert space on interval $[0, L], L>0$ represents the interval length, with $L_{2}$ norm

$$
\|\rho(x, t)\|_{2}=\left[\int_{0}^{L} \rho^{2}(x, t) \mathrm{d} x\right]^{1 / 2}, \begin{aligned}
& \rho(x, t) \in H^{2}(0, L) .
\end{aligned}
$$

For notational convenience, we denote

$$
\begin{aligned}
\rho_{t}(x, t) & =\frac{\partial \rho(x, t)}{\partial t}, \\
\rho_{x}(x, t) & =\frac{\partial \rho(x, t)}{\partial x}, \\
\rho_{x x}(x, t) & =\frac{\partial^{2} \rho(x, t)}{\partial x^{2}} .
\end{aligned}
$$

\section{Mathematical Modeling}

The Lighthill-Whitham-Richards (LWR) model [27, 28] based on the conservation law of mass is recommended in this paper to represent the crowd evacuation dynamics in one dimension, implying that the number of pedestrians coming in and going out of a corridor section account for the change of crowd density on that section. The LWR model is given by

$$
\rho_{t}(x, t)+q_{x}(x, t)=0, \quad(x, t) \in \Omega .
$$

where $\Omega=(0, L) \times(0,+\infty), x$ and $t$ are space and time variables, respectively. $\rho(x, t)$ is the average crowd density and $\rho_{t}(x, t)$ is the partial derivative of the density $\rho(x, t)$ with respect to time $t$ at position $x . q(x, t)$ denotes the flux of the crowd and $q_{x}(x, t)$ is the partial derivative of the flux $q(x, t)$ with respect to position $x$ at time $t$. The flux $q(x, t)$ is a function of $\rho(x, t)$ and the average crowd speed $v(x, t)$, as shown below,

$$
q(x, t)=\rho(x, t) v(x, t), \quad(x, t) \in \Omega .
$$

Various models have been developed to mimic the velocity-density relationship, such as Greenshield model, Drew model, Greenberg model, Pipes Munjal model, and Underwood model [29]. Here, the diffusion model is recommended, which is given as

$$
v(x, t)=v_{f}\left[1-\frac{\rho(x, t)}{\rho_{m}}\right]-\frac{D \rho_{x}(x, t)}{\rho(x, t)}, \quad(x, t) \in \Omega,
$$

where $v_{f}$ denotes the free flow speed, that is, the maximum moving speed when the density is zero. $\rho_{m}$ is the maximum crowd density, and $D$ denotes diffusion coefficient which is a positive constant, given by $D=\tau v_{r}^{2}$, where $v_{r}$ is a random velocity and $\tau$ is a relaxation parameter.

Remark 1. The diffusion model (5) is an extension of the Greenshield's model where the speed depends not only on the traffic density but also on the density gradient. The diffusion term demonstrates the fact that pedestrians can adjust their movement speed in real time based on the density ahead. The adjustment makes the change of their speed gradual rather than abrupt in response to the shock wave, which creates the dependence of speed on density gradient [29].

Combining the LWR model (3) with the diffusion model (5) yields

$$
\begin{aligned}
\rho_{t}(x, t)= & D \rho_{x x}(x, t) \\
& -\frac{\partial}{\partial x}\left[\rho(x, t)\left(1-\frac{\rho(x, t)}{\rho_{m}}\right) v_{f}\right],
\end{aligned}
$$


By choosing the free flow speed $v_{f}$ as the distributed control variable denoted by $\mathrm{u}(\mathrm{x}, \mathrm{t})$, one can derive

$$
\begin{aligned}
\rho_{t}(x, t)= & D \rho_{x x}(x, t) \\
& -\frac{\partial}{\partial x}\left[\rho(x, t)\left(1-\frac{\rho(x, t)}{\rho_{m}}\right) u(x, t)\right], \\
& \quad(x, t) \in \Omega,
\end{aligned}
$$

where $u(x, t) \in\left[-v_{m}, v_{m}\right]$ is the controller and $v_{m}$ is the maximum velocity. The model is subject to the following initial condition and boundary condition

$$
\begin{aligned}
\rho\left(x, t_{0}\right) & =\rho_{0}(x), \quad \forall x \in(0, L), \\
\rho(0, t) & =0, \\
\rho(L, t) & =0,
\end{aligned}
$$

$$
\forall t \in(0, \infty) \text {. }
$$

Remark 2. The paper [30] has suggested that pedestrian traffic can be handled in the similar way as the vehicle traffic. But there is a main difference between pedestrian traffic and vehicle traffic. In the vehicle traffic, the car on a lane is unidirectional, so its speed can be fixed by the traffic density, using the diffusion model. While in the pedestrian traffic people can move in both directions, its density cannot fix the speed with any velocity-density relationship model, so the free flow speed $v_{f}$ is chosen as the control variable, and, with the actuation system, people can be told to change their speed.

\section{Finite-Time Control}

In this section, by virtue of the finite-time control theory, a distributed controller is designed to make the state converge to zero in finite time. The stability of the crowd evacuation system under the finite-time controller is analyzed using the Lyapunov method.

The important finite-time control theory ([19], Lemma 4.3) is stated by the following lemma.

Lemma 3 (see [19]). Let an everywhere nonnegative function $W(t)$ meet the differential inequality

$$
\dot{W}(t) \leq-2 \gamma W^{\alpha}(t)
$$

for all $t \geq 0$ and for some constants $\gamma \geq 0$ and $\alpha \in(0,1)$. Then, $W(t)=0$ for all

$$
t \geq[2 \gamma(1-\alpha)]^{-1} W^{(1-\alpha)}(0) .
$$

The following lemma is an important inequality used in our proof, which can be considered as a special case of Hölder integral inequality.

Lemma 4 (see [31]). Consider an arbitrary real coefficient $p \geq$ 1 , and let $\rho(\cdot, t) \in L_{p}(0, L)$, where $L_{p}(0, L)$ is $p$-th integrable Banach space defined on interval $(0, L)$. Then the following inequality holds,

$$
\left[\int_{0}^{L}\left|\rho^{2}(x, t)\right| d x\right]^{p} \leq \int_{0}^{L}\left|\rho^{2}(x, t)\right|^{p} d x .
$$

In order to stabilize the crowd evacuation system (7) in finite time, the following distributed controller is designed,

$$
u(x, t)=\frac{\lambda_{1} \rho_{m}}{\rho_{m}-\rho(x, t)} \int_{0}^{x} \frac{\rho^{\alpha_{1}}(\xi, t)}{\|\rho(\xi, t)\|_{2}} \mathrm{~d} \xi, \quad(x, t) \in \Omega,
$$

where $\lambda_{1}$ is a constant control coefficient, $\alpha_{1} \in(0,1)$ is a constant.

Remark 5. The distributed control mentioned in [32, 33] means that the controller of a system can use information of the connected systems to construct the control strategy. However, the distributed controller mentioned in this paper is the controller of distributed parameter systems (as opposed to a lumped parameter system) whose state space is infinitedimensional.

Theorem 6. The crowd evacuation system (7), subject to the initial and boundary conditions (8), with the distributed controller (12), achieves the attainment of $\|\rho(x, t)\|=0$ in $L_{2}$ norm when $t \geq\left(2 / \lambda_{1}\left(1-\alpha_{1}\right)\right)\left(\int_{0}^{L} \rho_{0}^{2}(x) d x\right)^{\left(1-\alpha_{1}\right) / 2}$.

Proof. Consider the Lyapunov functional

$$
W(t)=\frac{1}{2} \int_{0}^{L} \rho^{2}(x, t) \mathrm{d} x, \quad t \geq 0 .
$$

Computing the time derivative of $W(t)$ for $t \geq 0$ yields

$$
\begin{gathered}
\dot{W}(t)=\int_{0}^{L} \rho(x, t) \rho_{t}(x, t) \mathrm{d} x=\int_{0}^{L} \rho(x, t) \\
.\left\{D \rho_{x x}(x, t)\right. \\
\left.-\frac{\partial}{\partial x}\left[\rho(x, t)\left(1-\frac{\rho(x, t)}{\rho_{m}}\right) u(x, t)\right]\right\} \mathrm{d} x .
\end{gathered}
$$

Substituting the controller (12) into (14) for $t \geq 0$ and using the Leibniz integral rule

$$
\begin{aligned}
& \frac{d}{d x}\left(\int_{a(x)}^{b(x)} f(x, t) d t\right) \\
& =f(x, b(x)) \frac{d}{d x} b(x)-f(x, a(x)) \frac{d}{d x} a(x) \\
& \quad+\int_{a(x)}^{b(x)} \frac{\partial}{\partial x} f(x, t) d t,
\end{aligned}
$$


one can derive

$$
\begin{aligned}
& \dot{W}(t)=\int_{0}^{L} \rho(x, t)\left\{D \rho_{x x}(x, t)\right. \\
& -\lambda_{1} \rho_{x}(x, t) \int_{0}^{x} \frac{\rho^{\alpha_{1}}(\xi, t)}{\|\rho(\xi, t)\|_{2}} \mathrm{~d} \xi \\
& \left.-\lambda_{1} \rho(x, t) \frac{\rho^{\alpha_{1}}(x, t)}{\|\rho(x, t)\|_{2}}\right\} \mathrm{d} x=D \int_{0}^{L} \rho(x, t) \\
& \cdot \rho_{x x}(x, t) \mathrm{d} x-\lambda_{1} \int_{0}^{L} \rho(x, t) \rho_{x}(x, t) \\
& \cdot \int_{0}^{x} \frac{\rho^{\alpha_{1}}(\xi, t)}{\|\rho(\xi, t)\|_{2}} \mathrm{~d} \xi \mathrm{d} x-\lambda_{1} \int_{0}^{L} \frac{\rho^{2+\alpha_{1}}(x, t)}{\|\rho(x, t)\|_{2}} \mathrm{~d} x .
\end{aligned}
$$

Integrating by parts the first term of (16) and considering the boundary condition (8),

$$
\begin{aligned}
& D \int_{0}^{L} \rho(x, t) \rho_{x x}(x, t) \mathrm{d} x \\
& \quad=\left.D \rho(x, t) \rho_{x}(x, t)\right|_{0} ^{L}-D \int_{0}^{L} \rho_{x}^{2}(x, t) \mathrm{d} x \\
& \quad=-D \int_{0}^{L} \rho_{x}^{2}(x, t) \mathrm{d} x, \quad t \geq 0 .
\end{aligned}
$$

As to the second term of (16), by using the same manipulations, one can derive

$$
\begin{aligned}
& \int_{0}^{L} \rho(x, t) \rho_{x}(x, t) \int_{0}^{x} \frac{\rho^{\alpha_{1}}(\xi, t)}{\|\rho(\xi, t)\|_{2}} \mathrm{~d} \xi \mathrm{d} x \\
& \quad=\left.\left[\rho^{2}(x, t) \int_{0}^{x} \frac{\rho^{\alpha_{1}}(\xi, t)}{\|\rho(\xi, t)\|_{2}} \mathrm{~d} \xi\right]\right|_{0} ^{L} \\
& -\int_{0}^{L}\left[\rho_{x}(x, t) \int_{0}^{x} \frac{\rho^{\alpha_{1}}(\xi, t)}{\|\rho(\xi, t)\|_{2}} \mathrm{~d} \xi\right. \\
& \left.+\rho(x, t) \frac{\rho^{\alpha_{1}}(x, t)}{\|\rho(x, t)\|_{2}}\right] \rho(x, t) \mathrm{d} x=-\int_{0}^{L} \rho(x, t) \\
& \cdot \rho_{x}(x, t) \int_{0}^{x} \frac{\rho^{\alpha_{1}}(\xi, t)}{\|\rho(\xi, t)\|_{2}} \mathrm{~d} \xi \mathrm{d} x-\int_{0}^{L} \frac{\rho^{2+\alpha_{1}}(x, t)}{\|\rho(x, t)\|_{2}} \mathrm{~d} x, \\
& \quad \rho^{2} \geq 0,
\end{aligned}
$$

that is,

$$
\begin{gathered}
\int_{0}^{L} \rho(x, t) \rho_{x}(x, t) \int_{0}^{x} \frac{\rho^{\alpha_{1}}(\xi, t)}{\|\rho(\xi, t)\|_{2}} \mathrm{~d} \xi \mathrm{d} x \\
=-\frac{1}{2} \int_{0}^{L} \frac{\rho^{2+\alpha_{1}}(x, t)}{\|\rho(x, t)\|_{2}} \mathrm{~d} x, \quad t \geq 0 .
\end{gathered}
$$

Substituting (17), (19) into (16), the time derivative of $W(t)$ for $t \geq 0$ becomes

$$
\begin{aligned}
\dot{W}(t)= & -D \int_{0}^{L} \rho_{x}^{2}(x, t) \mathrm{d} x-\frac{\lambda_{1}}{2} \int_{0}^{L} \frac{\rho^{2+\alpha_{1}}(x, t)}{\|\rho(x, t)\|_{2}} \mathrm{~d} x \\
= & -D \int_{0}^{L} \rho_{x}^{2}(x, t) \mathrm{d} x \\
& -\frac{\lambda_{1}}{2} \frac{\int_{0}^{L}\left[\rho^{2}(x, t)\right]^{\left(2+\alpha_{1}\right) / 2} \mathrm{~d} x}{\|\rho(x, t)\|_{2}} .
\end{aligned}
$$

As to the second term of (20), by virtue of Lemma 4, equation (20) can be rewritten in the form

$$
\begin{aligned}
\dot{W}(t) \leq & -D \int_{0}^{L} \rho_{x}^{2}(x, t) \mathrm{d} x \\
& -\frac{\lambda_{1}}{2}\left[\int_{0}^{L} \rho^{2}(x, t) \mathrm{d} x\right]^{\left(1+\alpha_{1}\right) / 2} \\
\leq & -\frac{\lambda_{1}}{2}\left[\int_{0}^{L} \rho^{2}(x, t) \mathrm{d} x\right]^{\left(1+\alpha_{1}\right) / 2} \\
= & -2^{\left(\alpha_{1}-1\right) / 2} \lambda_{1} W^{\left(\alpha_{1}+1\right) / 2}(t), \quad t \geq 0 .
\end{aligned}
$$

According to Lemma 3, when $t \geq\left(2 / \lambda_{1}(1-\right.$ $\left.\left.\alpha_{1}\right)\right)\left(\int_{0}^{L} \rho_{0}^{2}(x) \mathrm{d} x\right)^{\left(1-\alpha_{1}\right) / 2}, W(t)$ converges to zero, that is, the distributed controller (12) makes the crowd evacuation system (7)-(8) achieve the attainment of $\|\rho(x, t)\|=0$ in $L_{2}$ norm.

\section{Finite-Time Tracking Control}

In this section, a finite-time tracking controller is designed to make the crowd density $\rho(x, t)$ follow a given reference density $R(x, t)$.

Assumption 7. The reference density $R(x, t)$ is smooth enough and its spatial derivatives up to the second order are square integrable in $L_{2}$ norm. Also, it satisfies the initial and boundary conditions

$$
\begin{aligned}
& R(x, 0)=R_{0}(x), \quad \forall x \in(0, L), \\
& R(0, t)=0, \\
& R(L, t)=0,
\end{aligned}
$$

$$
\forall t \in(0,+\infty)
$$

Define the tracking error as $e(x, t)=\rho(x, t)-$ $R(x, t),(x, t) \in \Omega$. By using (7), the error dynamic is given as

$$
\begin{aligned}
e_{t}(x, t)= & D e_{x x}(x, t)+D R_{x x}(x, t)-R_{t}(x, t) \\
& -\frac{\partial}{\partial x}\left[\rho(x, t)\left(1-\frac{\rho(x, t)}{\rho_{m}}\right) u(x, t)\right], \\
& (x, t) \in \Omega,
\end{aligned}
$$


and the initial and boundary conditions are

$$
\begin{aligned}
& e(x, 0)=\rho_{0}(x)-R_{0}(x), \quad \forall x \in(0, L), \\
& e(0, t)=0, \\
& e(L, t)=0,
\end{aligned}
$$

$$
\forall t \in(0,+\infty)
$$

In order to stabilize the error dynamic systems (23) in finite time, the distributed controller is designed as

$$
\begin{array}{r}
u(x, t)=\frac{\rho_{m}}{\rho(x, t)\left(\rho_{m}-\rho(x, t)\right)}\left[\lambda_{2} \int_{0}^{x} \frac{e^{\alpha_{2}+1}(\xi, t)}{\|e(\xi, t)\|_{2}} \mathrm{~d} \xi\right. \\
\left.-\int_{0}^{x} R_{t}(\xi, t) \mathrm{d} \xi+D \int_{0}^{x} R_{x x}(\xi, t) \mathrm{d} \xi\right], \quad(x, t) \in \Omega,
\end{array}
$$

where $\lambda$ is a constant control coefficient and $\alpha \in(0,1)$ is a constant. Then, the following result is gotten.

Theorem 8. Consider the crowd evacuation system (7) with the initial and boundary conditions (8) and the reference density $R(x, t)$ satisfying Assumption 7. Then, the error dynamic systems (23) subject to the boundary conditions (24) can be stabilized to zero in $L_{2}$ norm with the distributed controller (25) when $t \geq\left(1 / \lambda_{2}\left(1-\alpha_{2}\right)\right)\left(\int_{0}^{L}\left(\rho_{0}(x)-R_{0}(x)\right)^{2} d x\right)^{\left(1-\alpha_{2}\right) / 2}$.

Proof. Consider the Lyapunov functional

$$
W_{e}(t)=\frac{1}{2} \int_{0}^{L} e^{2}(x, t) \mathrm{d} x, \quad t \geq 0 .
$$

Computing the time derivative of $W_{e}(t)$ for $t \geq 0$ yields

$$
\begin{aligned}
& \dot{W}_{e}(t)=\int_{0}^{L} e(x, t) e_{t}(x, t) \mathrm{d} x=\int_{0}^{L} e(x, t) \\
& .\left\{D e_{x x}(x, t)\right. \\
& -\frac{\partial}{\partial x}\left[\rho(x, t)\left(1-\frac{\rho(x, t)}{\rho_{m}}\right) u(x, t)\right]-R_{t}(x, t) \\
& \left.+D R_{x x}(x, t)\right\} \mathrm{d} x .
\end{aligned}
$$

Substituting the distributed controller (25) into (27) for $t \geq 0$, and using the Leibniz integral rule (15), one can derive

$$
\begin{aligned}
\dot{W}_{e}(t)= & \int_{0}^{L} e(x, t)\left\{D e_{x x}(x, t)-\lambda_{2} \frac{e^{\alpha_{2}+1}(x, t)}{\|e(x, t)\|_{2}}\right\} \mathrm{d} x \\
= & \int_{0}^{L} e(x, t) e_{x x}(x, t) \mathrm{d} x \\
& -\lambda_{2} \int_{0}^{L} \frac{e^{\alpha_{2}+2}(x, t)}{\|e(x, t)\|_{2}} \mathrm{~d} x
\end{aligned}
$$

As to the first term of (28), integrating it by parts and considering the boundary condition (24), the equation (28) can be written as

$$
\begin{aligned}
\dot{W}_{e}(t)= & -D \int_{0}^{L} e_{x}^{2}(x, t) \mathrm{d} x \\
& -\lambda_{2} \frac{\int_{0}^{L}\left[e^{2}(x,, t)\right]^{\left(\alpha_{2}+2\right) / 2} \mathrm{~d} x}{\|e(x, t)\|_{2}} .
\end{aligned}
$$

By virtue of Lemma 4, the time derivative of $W_{e}(t)$ for $t \geq 0$ is

$$
\begin{aligned}
\dot{W}_{e}(t) & \leq-\lambda_{2}\left[\int_{0}^{L} e^{2}(x, t) \mathrm{d} x\right]^{\left(\alpha_{2}+1\right) / 2} \\
& =-\lambda_{2}\left(2 W_{e}(t)\right)^{\left(\alpha_{2}+1\right) / 2} .
\end{aligned}
$$

According to Lemma 3, the error dynamic system (23) with boundary conditions (24) can be stabilized to zero when $t \geq$ $\left(1 / \lambda_{2}\left(1-\alpha_{2}\right)\right)\left(\int_{0}^{L}\left(\rho_{0}(x)-R_{0}(x)\right)^{2} \mathrm{~d} x\right)^{\left(1-\alpha_{2}\right) / 2}$.

Remark 9. In the application of crowd evacuation, the crowd density can be stabilized to different values to achieve different control objectives, such as maximizing the evacuation flow and maximizing the pedestrian movement speed. Therefore, the research of tracking control is of great practical significance. Meanwhile, estimating the time when the crowd density stabilizes to the reference density can evaluate the effectiveness of the control strategy.

\section{Simulation Results}

In this section, numerical results are given to illustrate the effectiveness of the finite-time controller (12) and the finitetime tracking controller (25), respectively. The numerical method is the finite volume method. For simulation, the initial density is given by $\rho(x, 0)=G \exp \left(-(x-a)^{2}\right)$, with $a=2$ being the center of the Gaussian distribution and $G=4.8$ being the highest magnitude of the distribution. The constant reference density is $R_{c}=2.5$ and a general reference density is given by $R=2.5+\sin (0.5 \pi x) \cos (\pi t)$. The main parameters are $L=4, D=0.1, \lambda_{1}=2, \alpha_{1}=0.8, \lambda_{2}=$ 1.3, $\alpha_{2}=0.3, \rho_{\max }=5, v_{m}=1.4$.

When the controller $u(x, t)=0$, the mathematical model of the crowd evacuation system (7) shown in Figure 1 is a diffusion model $\rho_{t}(x, t)=D \rho_{x x}(x, t)$.

Figure 2 illustrates the density response of the crowd evacuation system with the finite-time controller (12). Because of the effect of the advection term, the density profile moves towards the exit $(x=4)$ and converges to zero in finite time. For a clearer demonstration, the density evolutions at $x=2$ and $x=4$ are shown in Figure 3. The density at $x=2$ becomes zero at about 6.1 seconds, the density at $x=4$ becomes zero 


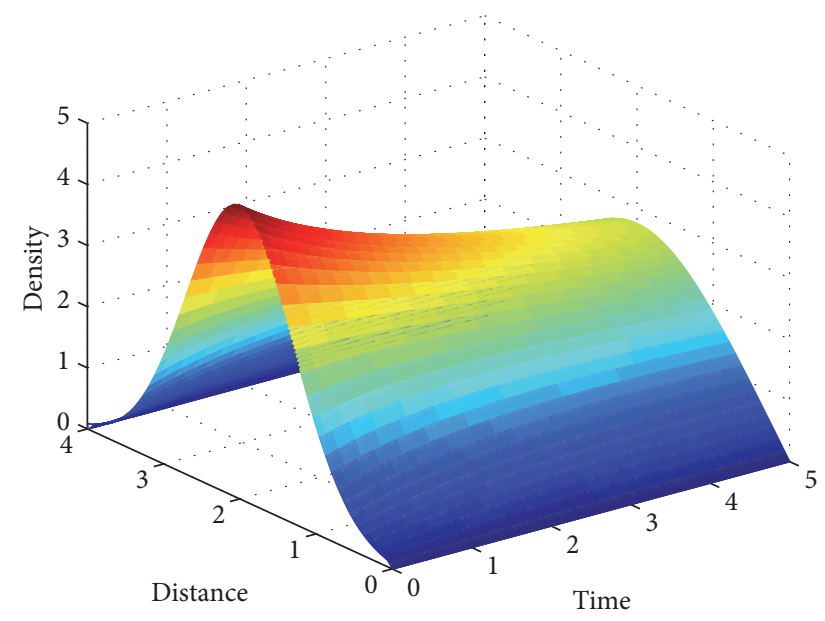

Figure 1: Density response of the uncontrolled crowd evacuation system.

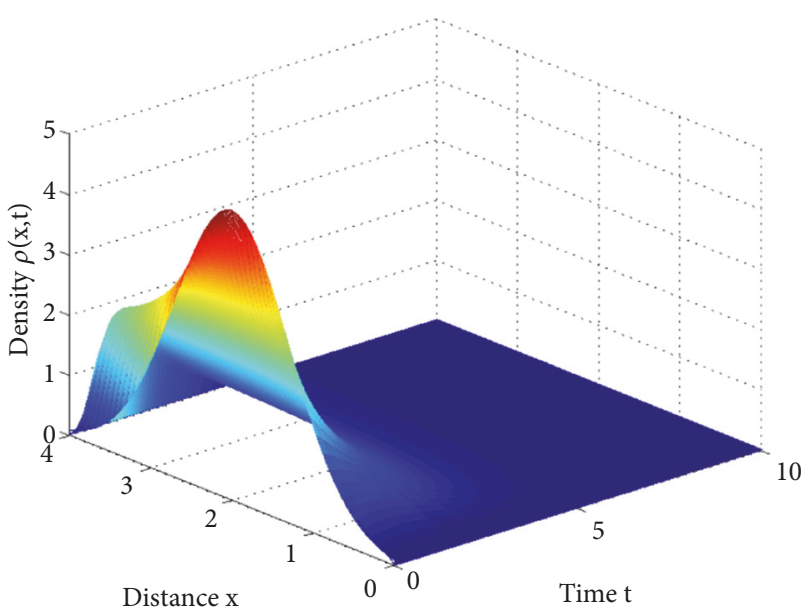

FIGURE 2: Finite-time control of the crowd evacuation system.

at about 7.1 seconds, and the crowd evacuation process ends. The evacuation time calculated by Theorem 6 is

$$
\begin{aligned}
t \geq & \frac{2}{\lambda_{1}\left(1-\alpha_{1}\right)}\left(\int_{0}^{L} \rho_{0}^{2}(x) \mathrm{d} x\right)^{\left(1-\alpha_{1}\right) / 2} \\
& =\frac{2}{2 *(1-0.8)}\left(\int_{0}^{L}(4.8\right. \\
& \left.\left.* \exp \left(-(x-2)^{2}\right)\right)^{2} \mathrm{~d} x\right)^{(1-0.8) / 2}=6.9987
\end{aligned}
$$

It can be easily seen that the calculated evacuation time and the simulated evacuation time are almost equal, and the error is within a reasonable range.

Figure 4 demonstrates the density response of the crowd evacuation system with the finite-time tracking controller (25), where $R(x, t)=2.5$ is chosen as the reference density. The density evolutions of $x=1$ and $x=2$ are shown in Figure 5 . It can be seen that the density at $x=1$ converges
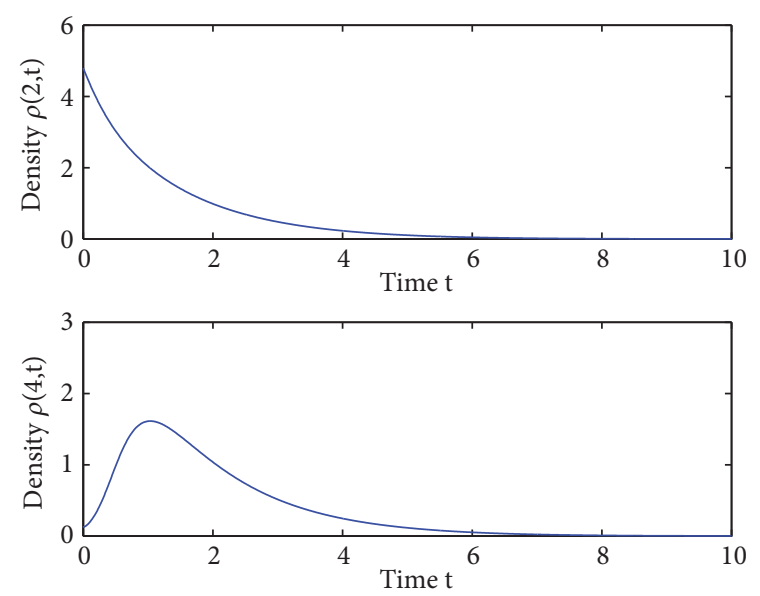

FIgURE 3: Density evolution at $x=2$ and $x=4$ with the finite-time controller.

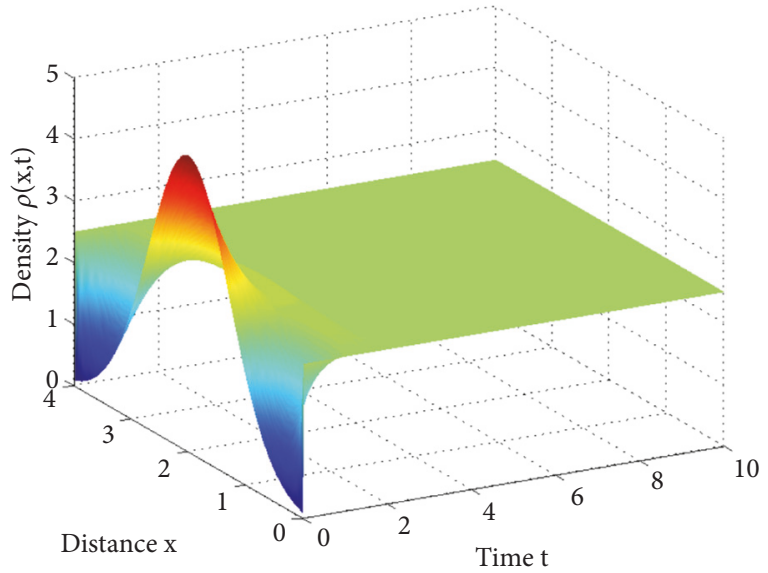

FIGURE 4: Finite-time tracking control with constant reference $R_{c}=$ 2.5.

to 2.5 at about 1.9 seconds and the density at $x=2$ converges to 2.5 at about 2.2 seconds. The evacuation time calculated by Theorem 8 is

$$
\begin{aligned}
t \geq & \frac{1}{\lambda_{2}\left(1-\alpha_{2}\right)}\left(\int_{0}^{L}\left(\rho_{0}(x)-R_{0}(x)\right)^{2} \mathrm{~d} x\right)^{\left(1-\alpha_{2}\right) / 2} \\
& =\frac{1}{1.3 *(1-0.3)}\left(\int _ { 0 } ^ { L } \left(4.8 * \exp \left(-(x-2)^{2}\right)\right.\right. \\
& \left.-2.5)^{2} \mathrm{~d} x\right)^{(1-0.3) / 2}=2.5861 .
\end{aligned}
$$

There is a small error between the calculated evacuation time and the simulated evacuation time, which may be caused by the discretization of simulation, but it is within a reasonable error range.

Next, a more general reference density $R=2.5+$ $\sin (0.5 \pi x) \cos (\pi t)$ is selected to show the effectiveness of the finite-time tracking controller, as shown in Figure 6.

The density response of the crowd dynamic system under the finite-time tracking controller is demonstrated in 

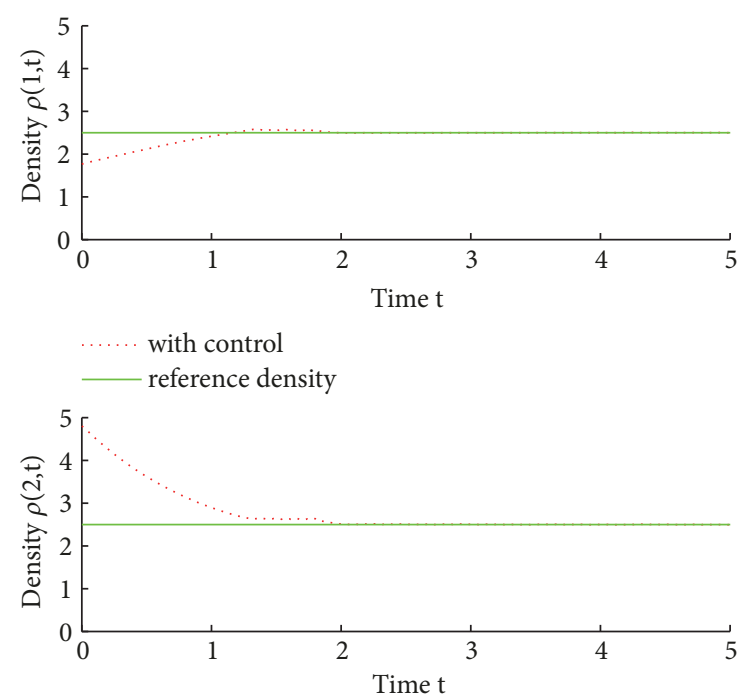

with control

reference density

Figure 5: Density evolution at $x=1$ and $x=2$ with Constant Reference $R_{c}=2.5$.

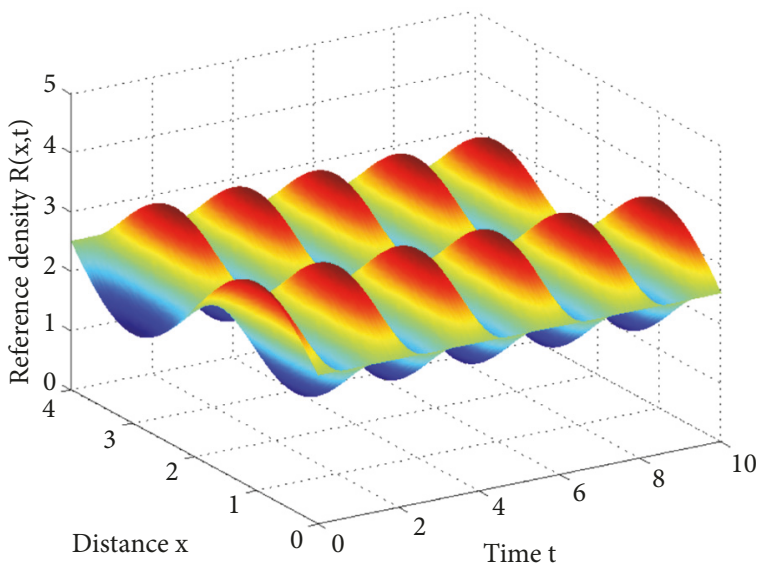

FIGURE 6: Reference density $R=2.5+\sin (0.5 \pi x) \cos (\pi t)$.

Figure 7. Figure 8 illustrates the density evolution at $x=1$ and $x=2$ with reference $R=2.5+\sin (0.5 \pi x) \cos (\pi t)$. The density at $x=1$ reaches the reference density at about 2.0 seconds and the density at $x=2$ reaches the reference density at about 2.9 seconds. Comparing the evacuation time calculated by Theorem 8 ,

$$
\begin{aligned}
t \geq & \frac{1}{\lambda_{2}\left(1-\alpha_{2}\right)}\left(\int_{0}^{L}\left(\rho_{0}(x)-R_{0}(x)\right)^{2} \mathrm{~d} x\right)^{\left(1-\alpha_{2}\right) / 2} \\
& =\frac{1}{1.3 *(1-0.3)}\left(\int _ { 0 } ^ { L } \left(4.8 * \exp \left(-(x-2)^{2}\right)\right.\right.
\end{aligned}
$$

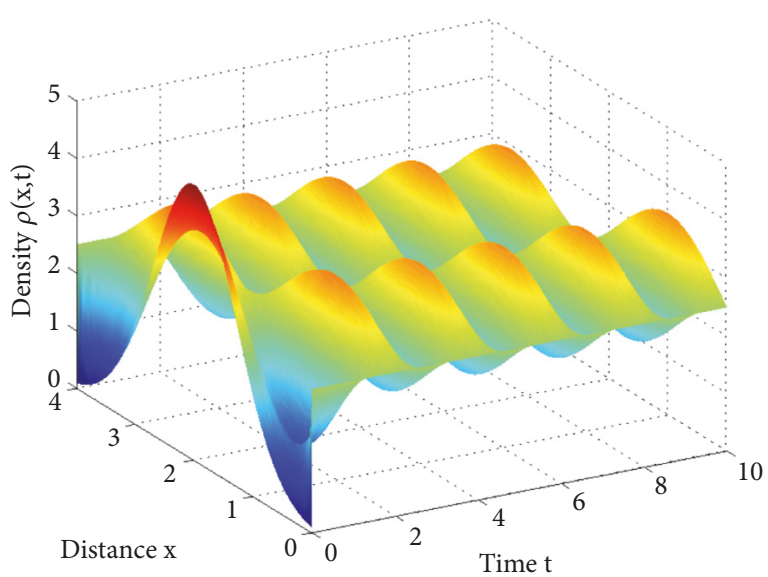

FIGURE 7: Finite-time tracking control with reference $R=2.5+$ $\sin (0.5 \pi x) \cos (\pi t)$.
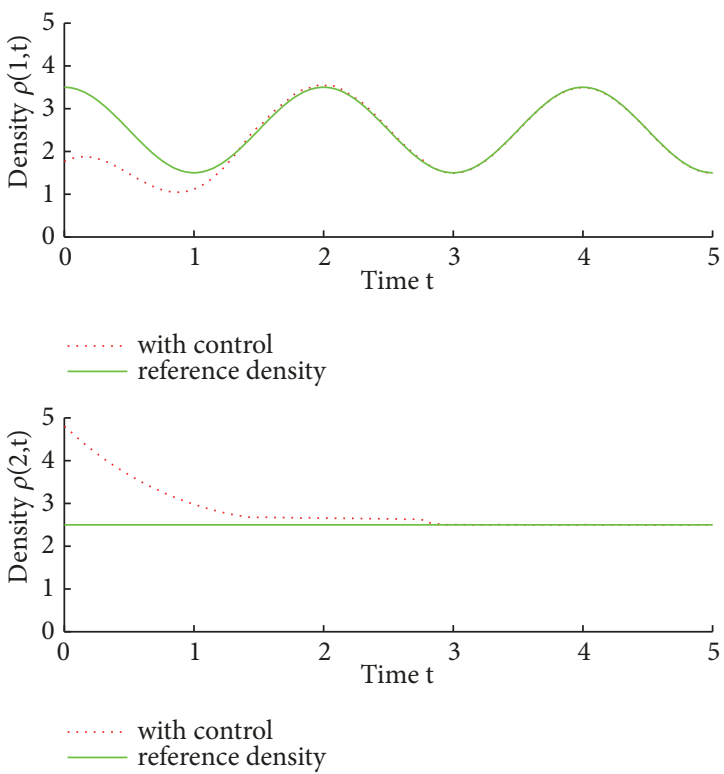

Figure 8: Density evolution at $x=1$ and $x=2$ with reference $R=2.5+\sin (0.5 \pi x) \cos (\pi t)$.

$$
\begin{aligned}
& \left.-2.5-\sin (0.5 * p i * x))^{2} \mathrm{~d} x\right)^{(1-0.3) / 2} \\
& =2.7350
\end{aligned}
$$

and they are almost equal.

To sum up, the effectiveness of the designed controllers has been shown by the comparisons, and the calculated evacuation time is almost equal to the simulated evacuation time, so the estimated evacuation time mentioned in the theorem is feasible. 


\section{Conclusion}

In this paper, the crowd dynamic model was constructed by combining the LWR model and the diffusion model. Then, finite-time controllers were designed for the crowd evacuation system, which solved the problem of nondirectionality of crowd movement and got the estimation of evacuation time. This theoretical research can promote the improvement of practical application, but the effect of time delay and disturbance in implementation needs to be further studied.

\section{Data Availability}

All the data used to support the findings of this study are available from the corresponding author upon request. The email address of the corresponding author is weiqin@vip.jiangnan.edu.cn.

\section{Conflicts of Interest}

The authors declare that they have no conflicts of interest.

\section{Acknowledgments}

This work was supported by National Natural Science Foundation of China (grant number 61807016) and Postgraduate Research \& Practice Innovation Program of Jiangsu Province (grant number KYCX17_1460).

\section{References}

[1] R. A. Smith and J. F. Dickie, "Engineering for crowd safety," in Proceedings of the International Conference on Engineering for Crowd Safety, Elsevier, Amsterdam, The Netherlands, 1993.

[2] R. Guo, H. Huang, and S. Wong, "Collection, spillback, and dissipation in pedestrian evacuation: a network-based method," Transportation Research Part B: Methodological, vol. 45, no. 3, pp. 490-506, 2011.

[3] N. Wagner and V. Agrawal, "An agent-based simulation system for concert venue crowd evacuation modeling in the presence of a fire disaster," Expert Systems with Applications, vol. 41, no. 6, pp. 2807-2815, 2014.

[4] N. Chooramun, P. J. Lawrence, and E. R. Galea, "An agent based evacuation model utilising hybrid space discretisation," Safety Science, vol. 50, no. 8, pp. 1685-1694, 2012.

[5] A. Lachapelle and M. Wolfram, "On a mean field game approach modeling congestion and aversion in pedestrian crowds," Transportation Research Part B: Methodological, vol. 45, no. 10, pp. 1572-1589, 2011.

[6] R.-Y. Guo, H.-J. Huang, and S. C. Wong, "Route choice in pedestrian evacuation under conditions of good and zero visibility: experimental and simulation results," Transportation Research Part B: Methodological, vol. 46, no. 6, pp. 669-686, 2012.

[7] L. A. Pereira, L. H. Duczmal, and F. R. B. Cruz, "Congested emergency evacuation of a population using a finite automata approach," Safety Science, vol. 51, no. 1, pp. 267-272, 2013.

[8] K. Cao, Y. Q. Chen, D. Stuart, and D. Yue, "Cyber-physical modeling and control of crowd of pedestrians: a review and new framework," IEEE/CAA Journal of Automatica Sinica, vol. 2, no. 3, pp. 334-344, 2015.

[9] D. Helbing and A. Johansson, "Pedestrian, crowd and evacuation dynamics," in Extreme Environmental Events, pp. 697-716, Springer, New York, NY, USA, 2011.

[10] R. L. Hughes, "A continuum theory for the flow of pedestrians," Transportation Research Part B: Methodological, vol. 36, no. 6, pp. 507-535, 2002.

[11] L. Huang, S. C. Wong, M. Zhang, C.-W. Shu, and W. H. K. Lam, "Revisiting Hughes' dynamic continuum model for pedestrian flow and the development of an efficient solution algorithm," Transportation Research Part B: Methodological, vol. 43, no. 1, pp. 127-141, 2009.

[12] C. Appert-Rolland, P. Degond, and S. Motsch, "Two-way multilane traffic model for pedestrians in corridors," Networks and Heterogeneous Media, vol. 6, no. 3, pp. 351-381, 2011.

[13] F. S. Hänseler, M. Bierlaire, B. Farooq, and T. Mühlematter, "A macroscopic loading model for time-varying pedestrian flows in public walking areas," Transportation Research Part B: Methodological, vol. 69, pp. 60-80, 2014.

[14] M. Twarogowska, P. Goatin, and R. Duvigneau, "Comparative study of macroscopic pedestrian models," Transportation Research Procedia, vol. 2, pp. 477-485, 2014.

[15] S. A. Wadoo, "Sliding mode control of crowd dynamics," IEEE Transactions on Control Systems Technology, vol. 21, no. 3, pp. 1008-1015, 2013.

[16] S. A. Wadoo and P. Kachroo, "Feedback control of crowd evacuation in one dimension," IEEE Transactions on Intelligent Transportation Systems, vol. 11, no. 1, pp. 182-193, 2010.

[17] H. Dong, X. Yang, Y. Chen, and Q. Wang, "Pedestrian evacuation in two-dimension via state feedback control," in Proceedings of the 2013 1st American Control Conference, ACC 2013, pp. 302-306, USA, June 2013.

[18] W. Qin, B. Zhuang, and B. Cui, "Boundary control of the crowd evacuation system based on continuum model," Control and Decision, vol. 33, no. 11, pp. 2073-2079, 2018.

[19] Y. V. Orlov, Discontinuous Systems-Lyapunov Analysis and Robust Synthesis Under Uncertainty Conditions, SpringerVerlag, Berlin, Germany, 2009.

[20] G. Zhang, C. Huang, X. Zhang, and W. Zhang, "Practical constrained dynamic positioning control for uncertain ship through the minimal learning parameter technique," IET Control Theory \& Applications, vol. 12, no. 18, pp. 2526-2533, 2018.

[21] X. Zhao, X. Wang, S. Zhang, and G. Zong, "Adaptive neural backstepping control design for a class of nonsmooth nonlinear systems," IEEE Transactions on Systems, Man, and Cybernetics: Systems, pp. 1-12, 2018.

[22] L. Ma, X. Huo, X. Zhao, B. Niu, and G. Zong, "Adaptive neural control for switched nonlinear systems with unknown backlash-like hysteresis and output dead-zone," Neurocomputing, vol. 357, pp. 203-214, 2019.

[23] X. Chang, R. Huang, and J. Park, "Robust guaranteed cost control under digital communication channels," IEEE Transactions on Industrial Informatics, pp. 1-9, 2019.

[24] X. Chang, R. Liu, and J. H. Park, "A further study on output feedback Ho control for discrete-time systems," IEEE Transactions on Circuits and Systems II: Express Briefs, pp. 1-1, 2019.

[25] G. Zhang, Y. Deng, W. Zhang, and C. Huang, "Novel DVS guidance and path-following control for underactuated ships in presence of multiple static and moving obstacles," Ocean Engineering, vol. 170, pp. 100-110, 2018. 
[26] X. Zhao, X. Wang, L. Ma, and G. Zong, "Fuzzy-approximationbased asymptotic tracking control for a class of uncertain switched nonlinear systems," IEEE Transactions on Fuzzy Systems, pp. 1-1, 2019.

[27] M. J. Lighthill and G. B. Whitham, "On kinematic waves: I. flow movement in long rivers. ii. a theory of traffic flow on long crowded roads," Pharmacology \& Therapeutics, vol. 53, no. 3, pp. 317-345, 1955.

[28] P. I. Richards, "Shock waves on the highway," Operations Research, vol. 4, no. 1, pp. 42-51, 1956.

[29] P. Kachroo, K. M, and K. M. Özbay, Feedback Ramp Metering in Intelligent Transportation Systems, Springer Science \& Business Media, 2011.

[30] A. C. May, Traffic Flow Fundamental, PrenticeHall, Englewood Cliffs, NJ, USA, 1990.

[31] M. Abramowitz and I. A. Stegun, Handbook of Mathematical Functions, with Formulas, Graphs, and Mathematical Tables, Dover, New York, NY, USA, 1972.

[32] X. Jin, S. Wang, G. Yang, and D. Ye, "Robust adaptive hierarchical insensitive tracking control of a class of leader-follower agents," Information Sciences, vol. 406-407, pp. 234-247, 2017.

[33] X. Jin, S. Wang, J. Qin, W. . Zheng, and Y. Kang, "Adaptive faulttolerant consensus for a class of uncertain nonlinear secondorder multi-agent systems with circuit implementation," IEEE Transactions on Circuits and Systems I: Regular Papers, vol. 65, no. 7, pp. 2243-2255, 2018. 


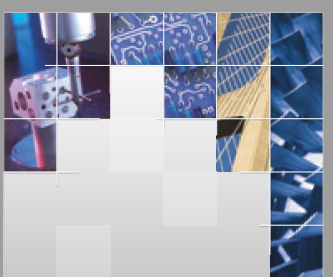

\section{Enfincering}
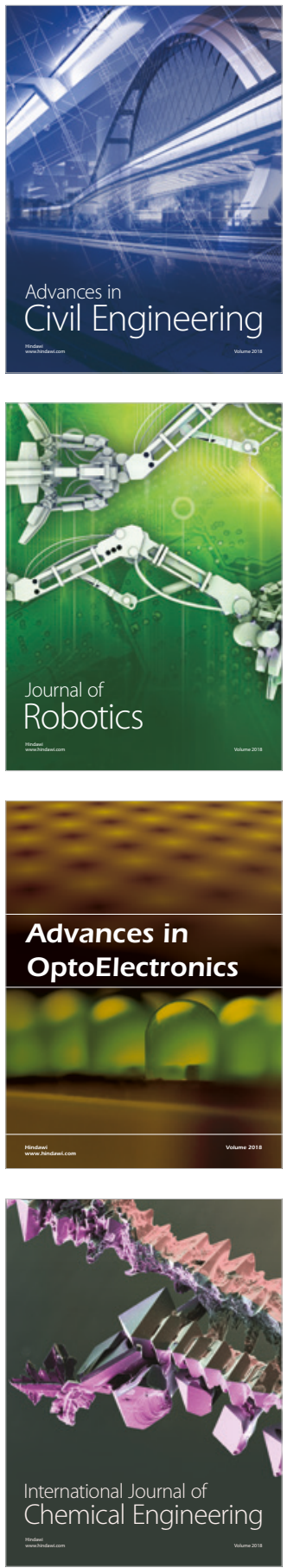

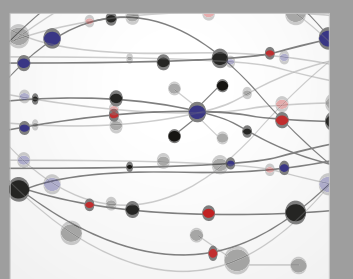

\section{Rotating \\ Machinery}

The Scientific World Journal

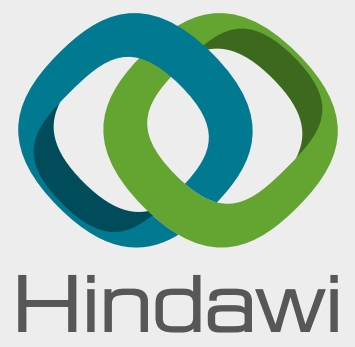

Submit your manuscripts at

www.hindawi.com
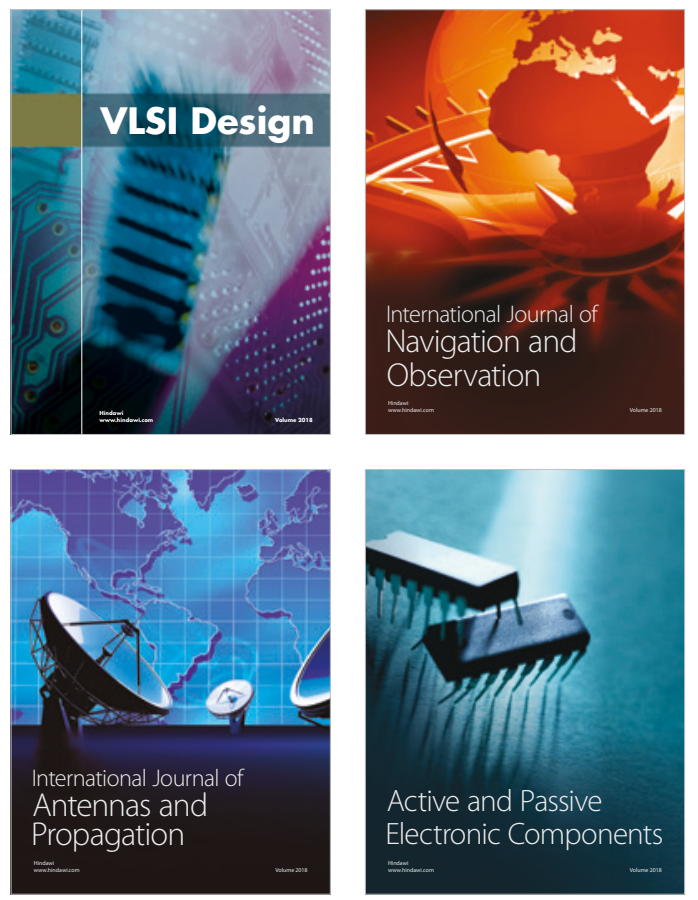
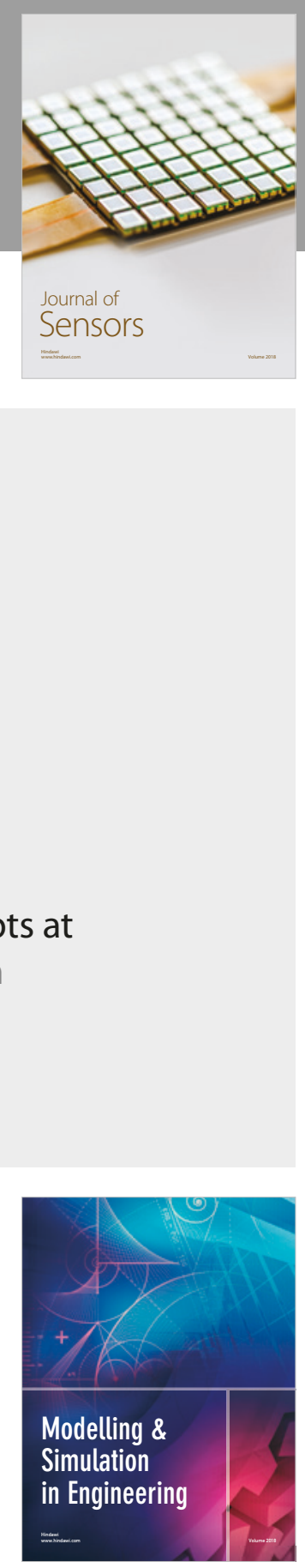

\section{Advances \\ Multimedia}
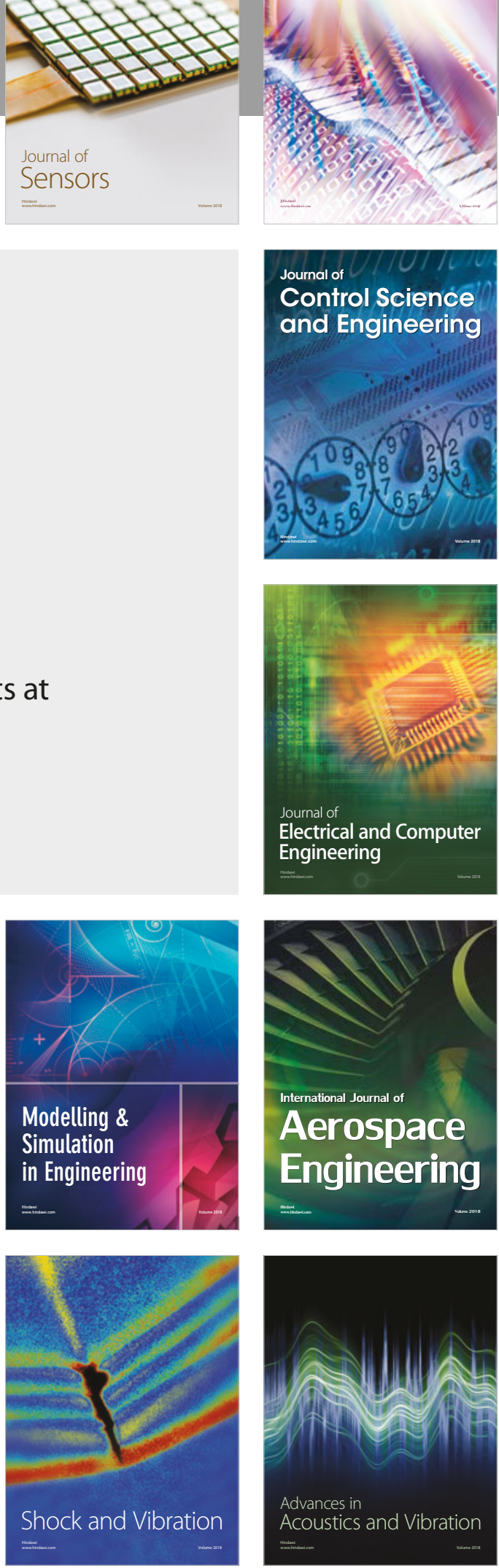